\section{Obtenir facile}

Obtenir facilement, sans difficulté, sans effort, sans peine

Transitif

1865 Voulez-vous jouir d'un magnifique panorama mouvant? vous l'obtenez facile, sans fatigue et vraiment enchanteur en prenant le chemin de fer de Liége à Aix-la-Chapelle par Maestricht avec retour par les prairies du Limbourg et les bords de la Vesdre (Albert d'Otreppe de Bouvette, Tout ce qu'on voudra)

1962 À Versailles, il [= un commerçant] en $o b$ tiendrait facile, facile le triple (Exemple entendu, 19 août 1962 / Grundt : 219)

\section{CORPUS WEB :}

Et pleins de petites choses optionnelles comme des bannières, des " posters » (enfin des gravures je sais pas un truc qui met en scène nos aventures ou les aventures des héros de la horde/alliance (ca pourrait s'obtenir facile par une suite de quêtes, ou archéo tiens, avec l'archéo on pourrait avoir pleins de trucs sympas à exposer, il y a souvent genre des décos style récipients/vase même à poser vite fait sur une étagère en bois) [http://eu.battle.net/wow/fr/forum/ topic/12844174943] (13.8.2015)

La silice et microballon sont complémentaires, je connaissais déjà les microballons mais la silice c'est vraiment très intéressant pour obtenir facile une pate, facile à travailler [http://www. shaperoom.net/forum/viewtopic.php?f=13\&t=96 22\&hilit=\&start=15] (13.8.2015)

$\mathrm{Tu}$ pourras aller sur le mien et mettre des comms, et tu pourras mefaire de la pub (mé t pô obligé) j'ai des amies qui mont dit qu'elles étaient aller sur ton blog, et ele l'on trouvé super super super bien (ex : zoubi-lov3 -> ou un truc comme ca...) et bravo, tu les a obtenus facile tes 1000 comm's, fallait pô demander, ils allaient bien finir par tomber^^ [http://x-still-th-x.skyrock.com/ 872062576-posted-on-2007-05-04.html] (13.8.2015)

kof $12=$ les trophés sont à chier, tu les obtiens faciles [http://www.jeuxvideo.com/forums/160-3822455-6-0-1-0-le-jeu-ou-les-trophees-sonthyper-facile.htm] (13.8.2015)

REMARQUES : Employé dans le langage familier, obtenir facile souligne l'acquisition d'une chose ou d'un bien à un bon prix et sans effort. Facile reste invariable dans la majorité des cas (v. le troisième exemple du $\mathrm{CW}$ ), mais dans le quatrième exemple du CW il s'accorde avec l'objet au pluriel tout en gardant son interprétation d'adverbe de manière. Notons l'emploi pronominal-impersonnel dans le premier exemple du CW. Relevons aussi la réduplication facile, facile.

\section{Odorer bon}

Dégager une odeur agréable

Intransitif

1888 Il restait pensif, pris d'une espèce de superstition pour ce sacerdoce, avec des regards en dessous vers Madeh, dont les cheveux odoraient bon (Jean Lombard, L'Agonie)

1902 Ceux qui sont nourris là-dedans, ne peuvent pas avoir le sens commun plus que ne peut odorer bon qui s'héberge en la cuisine (Pétrone, Satyricon [trad.])

1910 Un morceau de chair odorait bon parmi l'émiettement des mottes d'une taupinière. La bonne aventure ! Et, vlan ! Un coup de bec pour le déjeuner du matin (Louis Pergaud, De Goupil à Margot : histoire de bêtes)

2003 Ce sera bientôt la saison [...] des roses que mon épouse a importées d'Angleterre, tout cela devrait odorer bon. Hélas ! cela sentira quoi ? (Caroline et Jacques Bouchard, $L a$ Vie de château)

Transitif

1901 Tu me semblais rêver d'harmonies envolées, de rythmes plus subtils, de plus frêles chansons, indifférente aux gestes du chat familier qui, sous tes jupes soyeuses, odorant bon la femme (La Plume)

\section{Odorer doux}

Exhaler, répandre une odeur douce, délicate Intransitif

1325 Ainz ne fu d'arbre tiex dommages ; $\mathrm{Ne}$ fu espineus ne sauvages, Mais douz oudorans et souez (Watriquet de Couvin, Dits, p. 90, 225) 
REMARQUES : Odorer doux référait à l'odeur douce et délicate que dégage un objet (ici : un arbre). Notons la collocation avec soef.

\section{Odorer soef}

Exhaler, répandre une odeur suave, douce, agréable, délicieuse

Intransitif

+1365 Amours est le vin qui tant plaist, Odourant souef comme graine, Amours scet bien comment il est A ses gens et lesquels ont paine (Jean Froissart, Poésies [3 $3^{\mathrm{e}}$ tiers XIV $\mathrm{XI}^{\mathrm{e}}$ )

REMARQUES : Odorer soef s'appliquait à une chose qui dégage, émet des odeurs, des vapeurs d'une extrême douceur, produisant une impression agréable sur l'odorat.

\section{Fuvrer bel}

Bien agir, agir comme il faut

$\lambda$ raisonner bel

\section{Oloir bon}

Répandre une bonne odeur, une odeur agréable

Intransitif

1170 Jonchiez fu toz d'erbes dedenz, Que o les flors furent coillies : Ne furent flaistres ne mesties, Mout olurent buen e soëf [variantes : suef ; bon ; bien ; olant dolz et soëf ; flairoient] (Benoit de Sainte Maure, Le Roman de Troie, 13845)

REMARQUES : Usuel en ancien français, oloir bon renvoie à une chose, un élément de la nature (une fleur) qui émet, dégage des odeurs agréables. Notons la collocation avec soef qui ajoute à l'odeur délicieuse l'idée de doux parfum. Bon reste invarié.

\section{Oloir soef}

Répandre une odeur douce, délicate, agréable, délicieuse

Intransitif

1170a Soëf uelent, ços sai retraire, [variantes : flairent ; bien olant]

E si n'est color que n'i paire :
Ceus portent por le chaut d'esté (Benoit de Sainte Maure, Le Roman de Troie, 6231)

1170b Boche riant, levres grossettes Et un petitet vermeillettes Plus que samiz vermauz an grainne, Et plus soef oloit s'alainne Que pimanz ne basmes n’ançans ; Danz ot petiz, serrez et blans ; Manton et col, gorge et peitrine Ot plus blans que n'est nule ermine (Chrestien de Troyes [attribué], Philomena, 156)

REMARQUES : Le sujet de oloir soef désigne en ancien français une chose, un élément de la nature (une fleur) qui émet, dégage des odeurs agréables. Soef reste invariable et est modifié par plus.

\section{Opérer bas}

Agir, se conduire de manière immorale, libertine, sans souci des mœurs, sans scrupules Intransitif

1532 Donne ordre qu'ilz ne vivent en gentilz hommes, de leurs rentes, sans rien faire. Ne dea ! respondit Panurge, frere Jean, mon couillon gauche, je te croiray. Tu vas rondement en besoigne. Sans exception ne ambages tu m'as apertement dissolu toute craincte qui me povoit intimider. Ainsi te soit donné des cieulx, tousjours bas et roide operer (François Rabelais, Pantagruel)

\section{CORPUS WEB :}

Décembre - le ciel a fait de la terre son enclume, bas, très bas ; les arbres aux orées entrecroisent leurs cris. Incapable de s'insurger ni de soulever franchement la nuit, le jour passe, lourd comme un fer. A rendre l'âme. Il n'est que de s'asseoir, et de laisser passer sur soi ce fer, passer en soi la suie, laissez opérer tout bas a grâce de l'obscur, car en ce Temps l'obscur lui aussi a sa grâce [http://davidlerouge.fr/index. php?post/2010/12/15/respire] (13.8.2015)

Est ce que Monsieur Mavoungou a tord de dire haut ce que les pioniers de la division opèrent tout bas ?????? [http://www.i-services. com/membres/combox/combox.php?page $=1 \&$ uid $=131031 \&$ sid $=75786 \&$ id $=17935 \&$ iframe $=$ oui $]$ (13.8.2015) 
REMARQUes : Dans l'ancienne langue, opérer bas se dit du fait d'accomplir quelque chose, une action, le sujet adoptant un comportement vil, contraire à la morale ou aux bonnes mours, qui choque la bienséance par son caractère osé, licencieux. Notons la collocation opérer bas et raide, où raide se dit du fait d'exécuter quelque chose, une action d'une manière inconvenante, le sujet se montrant intransigeant, n'étant pas prêt à faire des compromis. Cette expression, qui est un terme emprunté au jeu de paume, porte, sous la plume de Rabelais, une connotation grivoise. Les citations du CW illustrent l'emploi moderne au sens de 'agir', bas pouvant prendre l'acception de 'en secret'. Bas et raide restent invariables. Notons l'emploi transitif du verbe dans le deuxième exemple du CW, où il réfère aux actions exécutées par un groupe (militaire). Notons le contraste dans dire haut ce que d'autres opèrent tout bas, calqué sur dire tout haut ce que d'autres pensent tout bas.

\section{Opérer raide}

Accomplir quelque chose, agir sèchement de manière rigide et inflexible

$\pi$ opérer bas

\section{Organiser dur}

Organiser avec énergie, en peinant

Pronominal

1959 Et pendant ce temps-là, ça s'organise dur, ferme et précis, du côté que vous savez. J'ai entre les mains un article paru dans le « Foyer rural », intitulé : « Comment le maire peut-il interdire un spectacle dans sa commune? " Petit traité de guerre civique prématuré (Canard enchaîné, 16 décembre 1959 / Grundt : 243)

\section{Corpus Web :}

Comment lui organiser son enterrement de vie de garçon alors que je suis une femme et donc ne serai pas de la soirée. C'est quelqu un qui est très entouré de filles et peu de gars dur pour léguer sans compter que $\mathrm{j}$ ai pas envie de léguer. Ca ne me dérange pas de ne pas être la le jour $j$ car c'est un moment pour eux mais je tiens a lui organiser dur dur [http://forum.aufeminin.com/ forum/f1255/_f1289_f1255-Je-suis-la-temoin-du- marie-comment-organiser-son-enterrement-devie-de-garcon.html] (13.8.2015)

Plusieurs posts arriveront donc dans les prochaines semaines directement sur le site (va falloir s'organiser dur !) [http://blog.ramenos. net/general/une-annee-chargee-un-blog-qui-vacontinuer-de-vivre-mais/] (13.8.2015)

c'était pour cet été, pour les rencontres de l'imaginaire, on s'organise dur cette année !! [http://pharendole.canalblog.com/archives/ 2008/06/05/9458757.html] (13.8.2015)

Cet été, j'étais surtout à la recherche des premiers N700 ! la traque s'organisait dur ! [http://www.denshaotaku365.com/archives/ 2007/10/04/6422557.html] (13.8.2015)

REMARQUeS : Organiser dur réfère à la peine et aux efforts déployés pour préparer une manifestation ou un spectacle selon un plan précis. Notons les adjectifs-adverbes ferme et précis. Ferme renvoie à la préparation soigneuse d'une manifestation ou d'un spectacle, menée avec assurance et maîtrise, selon un plan rigoureux, tandis que précis renvoie à un plan précis, déployé en vue d'un résultat déterminé. Dur, ferme et précis restent invariables. Le groupe tend à l'emploi pronominal, mais l'emploi transitif n'est pas exclu (v. premier exemple du CW). Le sujet est souvent impersonnel (on s'organise; v. organiser ferme), y compris l'emploi pronominal au sens passif (ça s'organise). Notons la réduplication dur dur dans le premier exemple du CW.

\section{Organiser ferme}

Organiser beaucoup, avec énergie

Pronominal

CORPUS WeB :

Dommage que ce soit en concurrence avec Valflaunès (34), il va falloir s'organiser ferme, ça va saturer avec la fête des mères, ça devient stressant la vie d'un amateur de reconstitutions ; vivement lundi qu'on se repose ! [http://www. paleobox.fr/t1283-orgnac-dernier-week-end-demai-2010] (13.8.2015)

a ben voilà, ça s'organise ferme à ce que je vois !;) Tant mieux !!!! [https://www.flickr.com/ photos/el_mexicano/3322736032/] (13.8.2015)

Je vois que l'on s'organise ferme... J'espère que vous prendrez des photos souvenirs pour 
ceux qui ne pourront pas participer à cette « rencontre tajine »[http://www.jardinature.net/ forum/ftopic2253-0-asc-15.html\&sid=8c8e860d9 2d14e5f0dc10340900457f9] (13.8.2015)

\section{Organiser précis}

Organiser avec précision

Pronominal

CORPuS Web :

$\mathrm{Ah}$, départs et retours de vacances, ça se croise ce w-e ! Des Balances, des Béliers s'organisent précis, d'enfer. Des Cancers, des Capricornes seront un peu chahutés [http://www. francebleu.fr/info-service-horoscope-pronosticbon-plan/l-horoscope-de-martin/disons-que-desopportunites-pointent-le-bout-du-nez] (15.8.2015)

\section{Oublier net}

Oublier tout d'un coup, brutalement

Transitif

1837 J'ai oublié net de vous dire que Clara est venue me voir (Stendhal, Correspondance)

CoRpus Web :

Moi ça m'arrive des fois d'oublier net ce que j'allais dire quelques secondes avant de le dire [http://forum.doctissimo.fr/sante/cannabis/ petit-probleme-language-sujet_151557_1.htm] (15.8.2015)

Serai que tu 1 oublie le mieux serait que tu rencontres quelqu un ça te permettrai de loublier net [http://forum.aufeminin.com/forum/f343 /_f54522_f343-Amoureuse-d-une-aventure.html] (15.8.2015)

Même le papier choisi contribue pour moi à la réussite de ce livre. Un papier pas totalement blanc, une bonne odeur de livre qui me fait oublier net ma tablette, des photos mates encore plus belles dévorer des yeux [http://www. amazon.fr/TOURTES-ET-PATES-NOS-REGIONS/ $\mathrm{dp} / 2501081501$ ] (15.8.2015)

l'info vient nous faire oublier nette la catastrophe de l'avion A 330 entre Rio et Paris... [http:// www.jeuxvideo.com/forums/1-21297-244-1-0-1-0metal-gear-rising-airbus-360.htm] (15.8.2015)

REMARQUES : Oublier net désigne le fait de perdre de façon involontaire, brusque et complète le souvenir de quelque chose, de ne pas se rappeler un rendez-vous ou une rencontre fixée ou décidée au préalable ; l'adjectif-adverbe net insiste alors sur l'absence totale du souvenir. Net reste invariable dans la majorité des cas (v. troisième exemple du CW), mais le dernier exemple du CW s'accorde avec l'objet direct sans changer de fonction.

\section{Ouïr clair}

Entendre bien, clairement, distinctement Transitif

1170 Andromacha saut fors par l'us ; Plaint sei e crie a si hauz criz Que mout par sont de loinz oïz : El grant palais perrin de Troie N'i a si sort qui cler ne l'oie. [variante : qi bien]

Plorer lor fait de chaudes lermes (Benoit de Sainte Maure, Le Roman de Troie, 15500)

-1200 Tervagant ! se l'eusse, bien me fust encontré :

Cil feist la bataille vers l'amiral el pré. Mais [il] est en la cambre, qui bien les oi cler (Elie de Saint Gille [fin XII $]$ ], 1607)

+1313 Ensi le ceualier looient

Si que les dames cler l'ooient

(Jean de Condé, Poèmes [1313-1337], 340)

1365 Et dames et puchellez, sergant et bacheler, Mainnent si grant dolour, c'on les oï bien cler,

Parmi le maistre ville. Dont s'alèrent armer Chevalier, escuïer, et les chevaus monter (Li Romans de Bauduin de Sebourc, Chant VI, 783)

+1415 Hola ! hola ! Souspir, on vous oyt bien. C'est a ung sourt a qui il le fault faire : Retrayez vous et pensez de vous taire, Car Dangier oit si cler qu'il n'y fault rien (Charles d'Orléans, Poésies [ 1415-1440], II, Rondel CCCIX, p. 25)

Intransitif

1532 Voyez là une belle medaille de coqu. Panurge à cause de ses lunettes oyoit des oreilles beaucoup plus clair que de coustume (François Rabelais, Pantagruel)

CORPUS WEB :

Et comme je ne suis pas ici pour vous jouer du pipeau et encore moins du ruine-babines, inventons tout de go le mot pour y oür clair : " œnomusicalité ». Comme dans " vin et mu- 
sique » [http://www.ledevoir.com/art-de-vivre/ vin/321648/oenomusicalite] (15.8.2015)

REMARQUES : Dans l'ancienne langue, oür clair désignait le fait d'entendre ou de comprendre parfaitement son interlocuteur. Clair reste invariable et est modifié par bien, plus, si. Il est aujourd'hui vieilli mais peut être employé occasionnellement par plaisanterie dans l'usage moderne.

\section{Ouïr dur}

Entendre mal, être un peu sourd

Intransitif

1568 - Monsieur, dit Fouquet, il est devenu tout sourd ; au moins il oyt bien dur. Il faudrait parler bien hault si vous vouliez qu'il vous entendist.

- Et bien ! dit le procureur, je parlerai prou haut

(Bonaventure Des Périers, Les Nouvelles Récréations et joyeux devis)

REMARQUES : Oür dur désignait dans l'ancienne langue le fait d'entendre son interlocuteur avec difficulté, avec peine, d'avoir du mal à comprendre ce qu'il dit. Notons l'emploi de parler prou haut 'très haut' (parler haut). VoIR AUSSI : entendre dur

\section{Ouvrer bel}

Bien travailler, de manière efficace Intransitif

$\sim 1250$ Mes or vueil je par vous savoir La quele doit avoir l'anel! Je di que cele ouvra mout bel Qui moine fist de son seignor (Les Trois Dames qui troverent l'anel, 268)

1364 PANTHALEON. Sire, a qui le costé fendi Longis l'avugle un venredi, Dont il yssi et yaue et sanc Qui coula de vostre saint flanc, Sur ses mains, et si bel ouvra Que sa veue recouvra, Pour quoy il vous cria merci, Sire, aussi a cest homme ci Rendez la veue en ceste voie, Par quoy chascun congnoisse et voie Que sur touz estes seul vraiz Diex (Miracle de saint Panthaleon, 523)

REMARQUES : Ouvrer bel se disait du fait de fabriquer, de créer quelque chose, de façonner un objet avec beaucoup de soin et d'application. Beau adopte la forme neutre bel qui était employée pour les fonctions adverbiales. Il est modifié par les adverbes d'intensité moult, si.

\section{Ouvrer fort}

Travailler en se donnant beaucoup de mal Transitif

+1300 Plus après avynt qe, par le assent de un roy d'Engleterre, furent les portes de le chastel, qe treblées erent, ars e espris par feu que fust illumée de bacons e de grece, e la tour sur la porte ars dedens. E le halt tour q'est en le tierce bayl de chastel, que fort et bien ovrée fust qe home ne saveit à cele oure nul plus fort ne meylour, fust de grant partie abatu, a cele bayle à poy tote destruyt (Fouke le Fitz Waryn [début XIV'], p. 44)

Remarques : Ouvrer fort désignait le fait de mettre beaucoup d'ardeur, d'efforts dans la fabrication d'un objet (ici : la construction d'une tour). Fort reste invariable et est modifié par plus. Fort est coordonné avec bien. VoIR AUSSI : travailler dur / fort

\section{Ouvrir droit}

Donner directement (sur quelque chose) Intransitif

1275 De son lit ert levez droit a cel ajornant, Pour oür les oisiaus qui soëf vont chantant, Une fenestre ouvri droit devers Oriant, De France li remenbre, si en va souzpirant, Ce fu un petitet devant soleil levant (Adenet le Roi, Buevon de Conmarchis, 2432)

REMARQues : Ouvrir droit s'appliquait à l'ouverture d'une fenêtre qui donne directement sur quelque chose, sur un endroit, le champ visuel s'étendant horizontalement, vers un lieu précis. C'est pourquoi droit forme un groupe prépositionnel avec devers. Droit reste invariable. Notons l'emploi de chanter soef.

\section{Ouvrir grand}

I. Ouvrir complètement, largement, le plus possible

Transitif

1547 Mais quand nostre amoureux produit un baudrier bien clousté et en bon equip- 
page, les portes fermees luy sont ouvertes grandes comme à passer une charretee de foin, qui est le souverain remede, la clef de la besongne, la peautre du navire, le manche de la charrue (Noël Du Fail, Les Propos rustiques)

1735 Ah ! Ah ! dit Dubois, en la voyant ouvrir toute grande [= la porte] par un valet de chambre du vieux marquis, vous verrez que c'est lui qui vient vous faire ses adieux ; ma foi, il faut qu'il vous aime bien pour cela : en effet, un moment après il parut à quatre pas de la porte ouverte toute grande, et me dit, je viens sçavoir comment vous vous portez, et vous dire adieu (Charles de Mouhy, La Paysanne parvenue)

1746 - J’y consens, me répondit-elle, mais surtout ne te marie point ; je fais serment que tu ne garderas ta femme, que lorsqu'elle aura été quinze jours devant mes yeux tous grands ouverts sans que je l'aperçoive... (Claude-Henri de Fusée de Voisenon, Le Sultan Misapouf et la princesse Grisemine)

1836 Des montagnes aux arêtes vives et tranchées le dentellent brusquement par les bords, et le soleil accoudé sur une des plus hautes cimes ouvre tout grand son œil jaune de lion aux paupières dorées (Théophile Gautier, Mademoiselle de Maupin)

1862 le beau, c'est que mon effraction ne servait à rien, qu'il fallait la recommencer. Je m'étais trompé de porte vitrée : où était la mienne ? Toute grande ouverte, à deux pas de moi ! Que dites-vous de celle-là ? Hélas ! Cette petite histoire eût bien amusé votre L... (Jean-Jacques et André-Marie Ampère, Correspondance)

1867 Elle ne pouvait fermer les yeux ; une effrayante contraction les tenait grands ouverts, fixés sur le spectacle horrible de la lutte. Elle était rigide, muette.

- Thérèse! Thérèse! Appela de nouveau le malheureux qui râlait (Émile Zola, Thérèse Raquin)

1880 Près de lui, un tout jeune homme, de dixsept ans au plus, quelque échappé de col- lège, ouvrait très grands ses beaux yeux de chérubin. Fauchery eut un sourire en le regardant (Émile Zola, Nana)

1888 Léon Daudet peignait hier l'intérieur de Fournier, le gendre et le successeur de Ricord, un intérieur toujours en festoiement, où l'on ne parle, dans les raouts et les dîners, que des parties naturelles de l'homme et de la femme et où, dans les bals, les jeunes filles peuvent lire, dans des livres tout grands ouverts sur les tables, des titres comme celui-ci : Du bubon (Edmond et Jules de Goncourt, Journal)

190017 mai.

Type de maire de village. Des sabots, une culotte avec deux grandes pièces à chaque genou, la braguette grand'ouverte comme si c'était plus convenable (Jules Renard, Journal)

1913 Un profond silence s'était établi. Léopold frappa trois fois à la porte de la chapelle. L'Organe l'ouvrit toute grande... Lui aussi est magnifiquement vêtu (Maurice Barrès, La Colline inspirée)

1929 J'en ai entendu, allez. De quoi écrire des livres et des livres. Lui, quand il m'avait ouvert tout grand son vieux cœur, il s'endormait, un sourire de bienheureux aux lèvres. Je l'aimais au fond, ce cher Franfranz (René Crevel, Êtes-vous fous ?)

1936 Autour de lui, sur des pontons, dix grues, sept ou huit aspirateurs, se pressaient, s'agitaient. Il en supportait jusque sur ses ponts. Par les écoutilles grandes ouvertes, on voyait ces engins plonger des bennes béantes, ou de longs tubes articulés, comme d'immenses suçoirs (Maxence Van der Meersch, L'Empreinte du dieu)

1943 - J'ai compris, Monsieur Philippe, dit-elle [= Daisy].

Visiblement, elle rassemble ses forces, bien qu'elle affecte de se détendre, laisse tomber les bras, ouvre tout grands ses yeux merveilleux, ses yeux d'ange... (Georges Bernanos, Monsieur Ouine) 
1945a La chambre interdite, pleine de nuit, de cauchemar et d'une odeur de folie, était ouverte toute grande (Simone de Beauvoir, Le Sang des autres)

1945b Une femme en cheveux riait, la bouche grand ouverte (Jean-Paul Sartre, Le Sursis)

1949 Il appela à voix basse : - Charlot! Charlot! Longin! Longin! Pas de réponse. Il se leva et marcha en titubant de sommeil jusqu'à la porte. Elle était grande ouverte. Un homme se cachait dans l'ombre (Jean-Paul Sartre, La Mort dans l'âme)

1956 c'était encore moi qui étais assis le premier, au premier rang, mes grandes oreilles de paysan grand ouvertes (Jean Anouilh, Pauvre Bitos)

1958 le voile de l'Inde où l'arbre Humanité ouvrait grand les bras pour abriter [...] à la fois des hommes, des femmes, des singes, des oiseaux et de magiques corolles (Thyde Monnier, Je ne suis pas des vôtres)

1959 Il ouvrit grand la bouche comme pour se gonfler d'air (Lettres françaises, 12 mars 1959 / Grundt : 391)

Pronominal

1715 Il passa au travers des arbrisseaux et il aperçut la porte qu'ils cachaient. Il se présenta devant et dit : " Sésame, ouvretoi ; » et dans l'instant la porte s'ouvrit toute grande (Antoine Galland, Les Mille et une nuits)

1823 Ce n'est pas du tout cela, lui dit Sieyès, voyant sa méprise ; je vais vous mettre au fait. Il renferme huit cent mille francs !!! (et ses yeux s'ouvraient tout grands) dans notre magistrature directoriale, nous avions réfléchi qu'un directeur sortant de place pouvait fort bien rentrer dans sa famille sans posséder un denier (Emmanuel de Las Cases, Le Mémorial de Sainte-Hélène)

1835 Ne voilà-t-il pas que l'écuyer qui courait en avant des chevaux s'arrêta précisément devant la porte de monsieur le curé, où la voiture eut la bonté de s'arrêter aussi, et daigna s'ouvrir toute grande. Il n’y avait personne dedans (Alfred de Vigny, Servitude et grandeur militaires)

1872 et l'on arrivait enfin à une vaste chambre, à une sorte de belvédère bâti sur le toit, derrière l'hôtel, au-dessus du quai de Béthune. Elle était en plein midi. La fenêtre s'ouvrait si grande, que le ciel, avec tous ses rayons, tout son air, tout son bleu, semblait y entrer (Émile Zola, La Curée)

1886 Il lui arrivait d'entonner les Fillettes de Paimpol; ou bien, en balançant la tête et battant la mesure avec son pied, elle prenait :

Pour la pêche d'Islande, mon mari vient de partir,

Il m’a laissée sans le sou,

Mais... trala, trala la lou...

J'en gagne !

J'en gagne !...

Chaque fois, cela s'arrêtait tout court, en même temps que ses yeux s'ouvraient bien grands dans le vague en perdant toute expression de vie, - comme ces flammes déjà mourantes qui s'agrandissent subitement pour s'éteindre (Pierre Loti, Pêcheur d'Islande)

1893 Elle porta ses mains de squelette à ses tempes, comme si elle avait senti son crâne éclater. Sa bouche s'était ouverte toute grande, et il n'en sortit aucun son : l'effrayant tumulte qui montait en elle, lui paralysait la langue (Émile Zola, Le Docteur Pascal)

1901 Pour la première fois, je vois une ville entièrement fortifiée, comme au $\mathrm{Xv}^{\mathrm{e}}$ siècle. Le célèbre roman de Balzac s'ouvre tout grand dans ma mémoire, en contemplant cet adorable vestige, "ce magnifique joyau de la féodalité » (Léon Bloy, Journal 2: Quatre Ans de captivité à Cochons-surMarne)

19025 novembre.

En voyage. Une dame se décide à ouvrir un sac de brioches. Elle y prend d'abord des miettes - on ne voit rien - et les mets dans une petite bouche serrée, discrète, distinguée. Peu à peu, l'audace lui vient. Le sac s'ouvre tout grand, et les morceaux 
de brioche entrent dans la bouche comme dans un four. Une autre vieille dame lit un livre et sourit (Jules Renard, Journal)

1908 Les vagues monstrueuses se soulevaient et leurs mâchoires écumantes s'ouvraient toutes grandes sur le vieillard. Vingt fois des paquets de mer emplirent l'embarcation (Anatole France, L'Île des pingouins)

1927a Tout à l'heure, après le discours de la grand-mère, l'interrogatoire de la cuisinière et les constatations d'usage, quand les gendarmes sont partis, l'enfant s'est sentie presque jalouse de ces gros hommes moustachus, pour qui toute grande s'ouvrait la nuit (René Crevel, Babylone)

1927b Point ne sera besoin qu'elle fasse le tour, sept fois, d'une prison, pour que des murs qui s'étaient grands ouverts au sourire infini des flots, à l'inverse de ceux de la cité biblique, se reconstruisent, et de pierres si inexorablement jointes, que nulle Cynthia, nul revenant léger ne sauraient se glisser (René Crevel, Babylone)

1932 la poussée mystique, si elle s'exerce quelque part avec assez de force, ne s'arrêtera plus net devant des impossibilités d'agir ; elle ne sera plus refoulée sur des doctrines de renoncement ou des pratiques d'extase ; au lieu de s'absorber en elle-même, l'âme s'ouvrira toute grande à un universel amour. Or ces inventions et ces organisations sont d'essence occidentale (Henri Bergson, Les Deux Sources de la morale et de la religion)

1942 Voilà une image bien représentative de notre temps : au moment où les oreilles des musiciens modernes s'ouvrent toutes grandes aux délices de ces accents d'outretombe, les peintres modernes tournent en sens inverse le bouton de leur appareil et coupent l'émission... Il est juste de dire que les surréalistes se montrent plus groupés (André Lhote, La Peinture d'abord)

1950 « C'est idiot de se promener comme ça au nez des Boches! » Le vieux cheval a soulevé la tête, dressé l'oreille comme s'il m'écoutait. Mais ses naseaux s'ouvrent tout grands et ses jambes se mettent à trembler : un obus siffle au loin, franchit la vallée en ronronnant, et plante une colonne de fumée jaune au-dessous du Bois-Haut, à mi-pente (Maurice Genevoix, Ceux de 14)

1957 Avec eux, l'éventail des admirations et des modèles s'ouvre plus grand, - et ce n'est pas un de leurs moindres mérites que d'avoir appelé notre attention, en y fixant d'abord la leur, sur des formes d'art avant eux peu connues, voire dédaignées

(Bernard Dorival, Les Peintres $d u x X^{e}$ siècle)

Intransitif

1735 Nous nous entretenions Dubois et moi de ces choses, lorsque j'entendis du bruit à la porte : voyez qui c'est, dis-je à ma tante ! Ah! Ah! dit Dubois, en la voyant ouvrir toute grande par un valet de chambre du vieux marquis, vous verrez que c'est lui qui vient vous faire ses adieux (Charles de Mouhy, La Paysanne parvenue)

II. S'ouvrir, s'étendre sur un vaste espace Transitif

1911 Il faut voir que tout est consentement. Il vit que tout était consentement, et non seulement devant lui, mais encore plus loin, sur le lac grand ouvert. Elles sont déjà assoupies et disent : « Nous voici dans notre repos » (Charles-Ferdinand Ramuz, Aimé Pache, peintre vaudois)

III. Avoir un avenir prometteur, offrir de nombreuses possibilités, de nombreuses opportunités

Transitif

1920 PENSÉE. J'écoute ce que vous dites.

ORIAN. Et quand vous seriez misérable encore et autant que vous le croyez, nous sommes jeunes ! Et la vie est grande ouverte devant nous, celle-ci, et l'autre par derrière qui n'a aucune fin (Paul Claudel, Le Père humilié)

IV. Faire résonner de toute sa puissance Transitif

1949 Ô coq de cette nuit absolument semblable À celui qui leva le rideau de la fable De sa voix grande ouverte et, tout en se cherchant, 
Rouge comme sa crête élaborait son chant. Ebréché par les nuits rugueuses de l'histoire,

Tu me trouves l'oreille à travers la nuit noire

(Jules Supervielle, Oublieuse Mémoire)

\section{CORPus Web :}

María Inés Rodríguez veut ouvrir grand les portes du CAPC [http://www.sudouest. fr/2015/03/22/maria-ines-rodriguez-veut-ouvrirgrand-les-portes-du-capc-1867059-2780.php] (15.8.2015)

Audimat, la revue qui donne envie d'ouvrir grand ses oreilles [http://www.clique.tv/cliquesur-audimat-la-revue-qui-donne-envie-douvrirgrand-ses-oreilles/] (15.8.2015)

Il faut se laisser porter et ouvrir grands les yeux [http://www.ouest-france.fr/il-faut-selaisser-porter-et-ouvrir-grands-les-yeux-1321902] (15.8.2015)

REMARQUES : Dans la tradition, l'accord de grand avec l'objet direct du verbe ouvrir est systématique (mais on hésite entre : ouvrir les yeux tous / tout grands ; ex. de 1746). L'emploi actuel tend tout de même à l'invariabilité (ex. de 1958, 1959 et dans le CW). On peut justifier l'accord par la modification : dans ouvrir les yeux tout grands, on peut interpréter grand comme propriété des yeux résultant du fait de les ouvrir. Mais on peut y voir également un simple accord de l'adjectif-adverbe dans une fonction plutôt adverbiale. Ceci apparaît clairement dans l'emploi antéposé au participe : grand(-)ouvert, souvent accordé. Le modifieur étant censé être un adverbe quand il modifie un adjectif, il s'insère dans la longue tradition d'accord morphologique partagé par des portes larges ouvertes, des fleurs fraîches écloses, elle est toute grande, une femme nouvelle venue, etc. Du point de vue fonctionnel, l'accord du modifieur grand avec le modifié ouvert est tout à fait conforme aux règles de logique grammaticale, l'accord se faisant de la même façon dans une grande fenêtre ou elle arrive contente, donc entre modifieur et modifié. C'est donc le dogme de l'invariabilité de l'adverbe qui veut que l'adjectif-adverbe ne s'accorde pas à chaque fois que la fonction en question est considérée adverbiale par les grammairiens. Gougenheim ne s'est pas fourvoyé en arguant par rapport à l'accord de tout que l'incohérence de l'usage actuel est le résultat « d'un conflit entre le désir des grammairiens d'imposer l'invariabilité et l'usage linguistique qui maintenait l'accord » (Georges Gougenheim, Système grammatical de la langue française, Paris, 1938, pp.129-130). Citons aussi René Radouant: «Dans les adjectifs composés, chaque terme était traité par l'ancienne langue comme un adjectif. De là les formes : Portes grandes ouvertes, fleurs fraîches écloses. Les oreilles pures françaises (Montaigne). Le français moderne au contraire tend à considérer le premier terme comme un adverbe et à le laisser invariable: Une petite fille nouveau-née, court-vêtue, demi-morte» (Grammaire française. Paris, 1922, p. 145). Les exemples du CW montrent que l'usage actuel tend de plus en plus vers l'invariabilité. Grand est modifié par bien, si, tout, très. Notons l'emploi de s'arrêter net.

\section{Ouvrir large}

I. Ouvrir complètement, largement, dans toute sa grandeur, dans toute sa largeur

Transitif

1832 Il monta donc dans la tour septentrionale, tandis qu'en bas le bedeau ouvrait toutes larges les portes de l'église, lesquelles étaient alors d'énormes panneaux de fort bois couverts de cuir, bordés de clous de fer doré et encadrés de sculptures « fort artificiellement élabourées » (Victor Hugo, Notre-Dame de Paris)

1839 À travers la porte de l'Enfer, large ouverte et non gardée, Satan passe et trouve tout désolé à l'entour ; car ceux qui avaient été commis pour siéger là avaient abandonné leur poste, s'étaient envolés vers le monde (François de Chateaubriand, Le Paradis perdu)

1851 Tantôt je le [= Dieu] vois comme un œil infini, comme vous dites, ouvert plus large que le ciel sur ses œuvres, qu'il regarde en s'élargissant pour les embrasser à mesure qu'il les crée ! (Alphonse de Lamartine, Le Tailleur de pierre de Saint-Point)

1879 « Vas-y, va ! » D’un bout à l'autre de la place du Capitole, les yeux étaient large ouverts, et chaque oreille aux écoutes (Léon Cladel, Ompdrailles, le Tombeau-des-Lutteurs) 
1881a Déjà le judaïsme avait professé sur le même sujet des maximes relativement humaines. Il avait ouvert aussi large que possible la porte des affranchissements. L'esclavage entre Hébreux était fort adouci. Les esséniens et les thérapeutes allèrent plus loin (Ernest Renan, Marc Aurèle et la fin du monde antique)

1881b Sur ces confins perdus du christianisme, les dogmes les plus divers se mêlaient. La tolérance des gnostiques, leur prosélytisme ouvraient si larges les portes de l'église que tout y passait (Ernest Renan, Marc Aurèle et la fin du monde antique)

1883 L'une représente Hélène, debout, droite, se découpant sur un terrible horizon éclaboussé de phosphore et rayé de sang, vêtue d'une robe incrustée de pierreries comme une châsse ; tenant à la main, de même que la dame de pique des jeux de cartes, une grande fleur ; marchant les yeux larges ouverts, fixe, dans une pose cataleptique (Joris-Karl Huysmans, L’Art moderne)

1887 Les feux chauffaient tellement la pièce, qu'on laissait larges ouvertes les deux fenêtres et la porte, par lesquelles entrait la bonne odeur pénétrante des foins, fraîchement coupés (Émile Zola, La Terre)

1892 Enfin, il le [= le blessé] coucha sur le petit lit de fer, en face de la fenêtre, dominant Paris, qu'il ouvrit toute large, dans un besoin de grand air et de lumière (Émile Zola, La Débâcle)

1929 MERCURE. Pour assister à leurs ébats, je convoque et tous les dieux, et toi, Léda, qui as encore à apprendre, et vous, braves gens qui avez été dans cette journée à la fois le personnel subalterne de l'amour et de la guerre, écuyer, guerrier, et trompette ! Ouvrez larges vos yeux et qu'autour du lit, pour étouffer leurs cris, résonnent chants, musique et foudre (Jean Giraudoux, Amphitryon 38)

1934 Il est bien, il bâille de nouveau, il s'allonge sur l'autre coude. Il a une espèce de veste déchirée par derrière jusque entre les épaules ; et, large ouverte par devant, elle découvre sa poitrine qui est creuse, en même temps qu'il a une barbe dure au menton (Charles-Ferdinand Ramuz, Derborence)

Pronominal

1876 - Oui, un déjeuner de pensionnaire, dit méchamment le sénateur.

À ce moment, il y eut à la porte un grand froissement d'étoffes ; le battant s'ouvrit très large, et une femme entra, vêtue d'une robe si chargée de nœuds, de fleurs et de dentelles, qu'elle dut presser la jupe à deux mains, pour pouvoir passer (Émile Zola, Son Excellence Eugène Rougon)

1877 Au plafond, le gaz avait passé comme un badigeon de suie. Les deux fenêtres s'ouvraient si larges, que les ouvrières, sans quitter l'établi, voyaient défiler le monde sur le trottoir d'en face. Madame Lerat, pour donner l'exemple, arrivait la première (Émile Zola, L’Assommoir)

1924 Madame Lenoir pense à sa mère et à sa fille Mélanie. On suit de petits couloirs étroits. On arrive devant le portail armorié d'un jardin royal. Les deux battants lourds s'ouvrent larges (Marcel Jouhandeau, Les Pincengrain)

II. Avoir un avenir prometteur, offrir de nombreuses possibilités, de nombreuses opportunités

Pronominal

1868 C'était une pénétration complète de chair et de cœur. D'ailleurs, la vie s'ouvrait large et tranquille, les époux regardaient sans crainte devant eux. Quatre années de félicité les rassuraient contre toute secousse (Émile Zola, Madeleine Férat)

1907 Comme tout cela était loin ! À la pensée qu'il s'était évadé de ce passé maussade, une joie puissante l'envahit et sa poitrine se gonfla, aspirant l'air des sommets et les grands souffles aventureux. Comme la vie s'ouvrait large devant lui, aux côtés de cette créature splendide, qui était déjà sa femme de chair, dont la possession ne 
le lasserait jamais ! (Émile Moselly, Terres lorraines)

1942 le nombre d'apprentis que le maître peut recevoir s'ils sont de son lignage, de près ou de loin, n'est pas limité. La voie s'ouvre moins large aux autres. Si un maître peut prendre d'apprentis « privés » le nombre qui lui plait, il n'a droit, sauf exception, qu'à un seul apprenti " estrange »

(Edmond Faral, La Vie quotidienne au temps de saint Louis)

1963 il [= l'escroc] entre si bien dans la peau de son personnage, qu'il commet son premier abus de confiance presque inconsciemment. En toute innocence, un petit emprunt qui va de soi. Après, il n'a plus qu'à continuer, la route s'ouvre toute large devant lui. Il se grisera facile (Alphonse Boudard, La Cerise)

III. S'ouvrir, s'étendre sur un vaste espace Pronominal

1926 C'est l'accord de l'homme et de l'or, C'est un regard lié à la terre.

Au hasard une délivrance,

$\mathrm{Au}$ hasard l'étoile filante

Et l'éternel ciel de ma tête

S'ouvre plus large à son soleil,

À l'éternité du hasard

(Paul Éluard, Capitale de la douleur)

Transitif

1931 Les Allemands sont encore loin là-bas, derrière Steenvorde. Ici toute la terre est maintenant large ouverte. C'est tout nu (Jean Giono, Le Grand troupeau)

\section{CORPUS WEB :}

Cherchant à arrêter le temps, les danseurs déploient une vibration singulière pour retrouver le naturel du mouvement et ouvrir large l'horizon du hip hop avec une danse lancinante, envoûtante et ronde d'où émergent des scènes de vie singulières sur fond de musiques savamment arrangées [http://www.leprisme.agglo-sqy.fr/ programmation/detail/evenement/le-moulindu-diable/] (15.8.2015)

Elle souhaite ouvrir large les portes du thêâtre en proposant des spectacles en itinérance, un événement fédérateur durant l'été, un temps fort sur les écritures théâtrales d'un pays étranger, créer " les journées du Matrimoine » mettant en avant les écritures d'auteures trop mal connues et des lectures tous les mois pour les jeunes spectateurs... [https://www.actualitte. com/communiques/theatre/nomination-decarole-thibaut-a-la-direction-du-centre-drama tique-national-de-montlucon-auvergne/2068] (15.8.2015)

A 18h ce 6éme jour, lére dose de sulfate de magnésium (qui permet d'ouvrir large les canaux $\mathrm{du}$ foie) [http://www.lapauserelax.fr/Quelquesconseils.html] (15.8.2015)

Pour cela, le dialogue est permanent, pour répondre judicieusement aux attentes, ouvrir larges les perspectives et donner à nos jeunes les outils de leur prise en charge d'abord, et à la prise en charge de la destinée du pays, ensuite [https:// www.youtube.com/watch? $\mathrm{v}=\mathrm{ibH}$ H1Sds1l0] (15.8.2015)

REMARQUES : L'objet direct du groupe ouvrir large (I) désigne, dans la plupart des exemples, quelque chose que l'on peut ouvrir en grand, largement, nécessitant l'intervention de l'homme : une porte, une fenêtre, un battant de porte ; il peut également renvoyer à un vêtement. L'objet direct désigne également très souvent une partie $\mathrm{du}$ visage (les yeux, les oreilles, la bouche, les narines, le nez) ou du corps (la main, les doigts) que le sujet ouvre de toute sa grandeur. Il peut aussi, dans un emploi figuré et métaphorique, désigner le cœur, le sujet cherchant à exprimer ses sentiments à autrui. (II) réfère à la vie et souligne toutes les possibilités ou opportunités qu'elle offre à quelqu'un. Dans (III), le sujet réfère au ciel, à un astre ou à un élément de l'espace (voie, route), insistant sur l'étendue, l'impression d'infini. Large peut s'accorder avec le sujet ou avec l'objet, mais l'accord est moins systématique qu'avec ouvrir grand (v. l'exemple de 1879). Dans l'emploi adjectival du verbe (participe passé), on note dans la plupart des cas l'accord de l'adjectif-adverbe large et de l'adverbe d'intensité (tout) qui le modifie. Large reste invarié dans le deuxième exemple du CW, mais l'accord est réalisé dans le dernier. Voir commentaire détaillé sur l'accord des modifieurs d'adjectifs dans l'entrée ouvrir grand. Large est modifié par plus, tout. 